\title{
Characterization and Classification of Soils under Lower Brahmaputra Valley of Assam, India
}

\author{
Juri Das ${ }^{1}$, R. M. Karmakar², Danish Tamuly ${ }^{2}$, Ingudam Bhupenchandra ${ }^{3}$ \\ and Laishram Kanta Singh ${ }^{*}$
}

${ }^{I}$ Department of Bio-resource Management, Defence Research Laboratory, Tezpur, India

${ }^{2}$ Department of Soil Science Assam Agricultural University, Jorhatm India

${ }^{3}$ Krishi Vigyan Kendra Tamenglong, ICAR Manipur Centre, India

${ }^{4}$ Krishi Vigyan Kendra Imphal West, ICAR Manipur Centre, India

*Corresponding author

\section{Keywords}

Land uses,

Classification,

Characterization

Article Info

Accepted:

10 November 2019

Available Online:

10 December 2019

\section{A B S T R A C T}

An investigation was carried out to characterize and classify soils of different land uses of lower Brahmaputra valley of Assam. Seven soil profiles were collected from a contiguous area under acacia (P1), bamboo (P2), coconut (P3), sisoo (P4), teak (P5), paddy (P6) and jackfruit (P7) land uses at Horticultural Research Station, Kahikuchi. The soils under different land uses varied in morphological, physical and chemical properties. The hue of the soil was $10 \mathrm{YR}$, value ranged from 5-7 and chroma varied from 2-8. Few, fine, faint to many, coarse, prominent mottles with colours of 7.5 YR $6 / 8$ to 7.5 YR $7 / 8$ were observed in the subsurface horizons of all the studied land uses. Few to abundant Fe-Mn glaebules were observed in the subsurface horizons of P2 (bamboo), P5 (teak) and P6 (paddy). Soil textural classes varied from sandy clay loam to clayey and the structure was sub angular blocky except in surface horizon of P6 (paddy). The clay content increased with soil depth which indicated fairly development of the soils. Soil organic carbon was highest in the surface horizon of a profile and it decreased regularly with soil depth. Bulk density of the soils varied from 1.31 to $1.47 \mathrm{Mgm}^{-3}$ and particle density varied from 2.40 to $2.61 \mathrm{Mgm}^{-3}$. The soil $\mathrm{pH}$ was found to be in acidic range (4.7-5.8) and the EC was low $\left(0.01\right.$ to $\left.0.09 \mathrm{dSm}^{-1}\right)$. Free oxides of $\mathrm{Fe}$ and $\mathrm{Al}$ increased down the profile. Exchangeable $\mathrm{Ca}^{2+}$ was the dominant basic cation followed by $\mathrm{Mg}^{2+}, \mathrm{K}^{+}$and $\mathrm{Na}^{+}$. Exchangeable $\mathrm{Al}^{3+}$ was dominant acid cation. The CEC and ECEC of the soils were low varying from 5.18 to $10.3 \mathrm{cmol}$ $(\mathrm{p}+) \mathrm{kg}^{-1}$ soils and 2.97 to $5.32 \mathrm{cmol}(\mathrm{p}+) \mathrm{kg}^{-1}$ soils respectively. The base saturation of the soils varied from 35.9- 57.2\%. On the basis of morphological, physical and chemical properties, the soils of the study area were classified as Typic Dystrudepts except P6 (paddy) which was classified as Aeric Dystrudepts. 


\section{Introduction}

In India sixty per cent of the population is engaged in farming, with subsistence farming along with century old practices that influence the soil quality. The success of soil management to maintain soil quality depends on understanding the responses of soils to different land uses and different management practices over time. Few studies on impacts of land use in agricultural management practices indicated these as the major driver of spatial variability in soil physical-chemical properties, as well as soil biota communities (Tilman et al., 2002; Wallenius et al., 2011). Modern agricultural practices are site-specific and require detailed study of the area. The properties of a soil are the basic attributes that directly influence the soil response to any specified use. The yield attributes is directly dependent on the soil and its characteristics.

In order to adopt good management practices and remedial measures for various soils, a systematic study of the soils is highly essential. Sporadic information was available on the soils of lower Brahmaputra valley of Assam. Hence, the present investigation was taken up to characterize and classify the soils of lower Brahmaputra valley of Assam

\section{Materials and Methods}

The study was conducted in Horticultural Research Station (HRS), Kahikuchi located in Kamrup district in Lower Brahmaputra Valley Zone (LBVZ) of Assam. The research station is situated at $26^{\circ} 6.03^{\prime} \mathrm{N}$ latitude and $91^{\circ} 35.64^{\prime} \mathrm{E}$ longitude at an elevation of $64 \mathrm{~m}$ above mean sea level and covers an area of 42.50 ha. Major part of Kamrup district is covered by younger and older alluvium of Quaternary Period. The Assam Himalayas are comparatively younger than Assam plateau and consist mainly of gneiss, schists, slates, limestones, dolomites etc. This feature has given rise to mixed mineralogy of the soils (Chakravarty et al., 1978; Karmakar and Rao 1999b). The climate of the study area is humid sub-tropical. The mean annual rainfall of the area is $1566 \mathrm{~mm}$, major portion of which is received during April-September (1484 mm). Soil moisture control section (SMCS) of the study area remains moist in most part of the year and is not dry for 90 cummulative days or 60 consecutive days following the summer solstice and therefore it qualified for 'udic' moisture regime. The localized low lying areas where water stands for a considerable period of time during the rainy season, the ground water table remains high and gives rise to 'aquic' moisture regimes characterized by low chroma. The mean annual temperature of the area is $26{ }^{\circ} \mathrm{C}$. The mean summer and mean winter temperatures of the area are $28.9^{\circ} \mathrm{C}$ and $23.2^{\circ} \mathrm{C}$, respectively. The area qualifies for 'hyperthermic' temperature regimes as the mean annual soil temperature is more than 22 ${ }^{\circ} \mathrm{C}$ and difference between mean summer and mean winter soil temperatures is more than 6 ${ }^{\circ} \mathrm{C}$.

Seven soil profiles were collected from areas under acacia land use (P1), bamboo land use (P2), coconut land use (P3), sisoo land use (P4), teak land use (P5), paddy land use (P6) and Jackfruit land use (P7). Morphological features of each pedon were studied in the field following standard procedure (All India Soil and Land Use Survey Organization 1971). The soil samples were air dried, ground and passed through a $2 \mathrm{~mm}$ sieve. The sieved soil samples were stored in polythene bags and subsequently used for analysis of various soil parameters. The particle size analysis was done as per international pipette method with sodium hexameta phosphate as dispersion agent after removal of organic carbon by hydrogen peroxide treatment. Organic carbon was determined by wet combustion (WalkeyBlack procedure), $\mathrm{pH}$ was measured in 1:2.5 soil/water suspension using $\mathrm{pH}$ meter. 
Exchangeable bases were extracted by $1 \mathrm{M}$ NH4OAc ( $\mathrm{pH}$ 7.0). The Cation Exchange Capacity (CEC) was determined with distillation method. Diothonite extractable Fe and $\mathrm{Al}$ were determined by standard procedure given by Mehra and Jackson 1958. Soil physical parameters were determined by Keen- Raczkowski box method.

\section{Results and Discussion}

\section{Morphological characteristics}

The soils under investigation possessed hue of 10YR, value ranging from 5 to 7 and chroma ranging from 2 to 8 (Table 1). The hue of 10YR is due to more humid soil condition which is common in alluvial derived soils of Assam as reported by different workers (Chakraborty et al., 1984; Chakravarty et al., 1978; Dutta 1992a). The value and chroma of the soils were higher in the subsurface horizons indicating more development of colour soil colour. Chroma of 2 in the surface horizon of P6 under rice land use is indicative aquic condition during some part of the year. Few, fine, faint to many, coarse, prominent mottles with colours of $7.5 \mathrm{YR} 6 / 8$ to $7.5 \mathrm{YR}$ $7 / 8$ were observed in the subsurface horizons of all the studied land uses. Presence of mottles of redder hue and higher chroma in the subsurface horizons of all the studied soils might be attributed to seasonal hydromorphic conditions and fluctuating ground water leading to oxidation-reduction cycles. Presence of few to abundant Fe-Mn glaebules in the sub-surface horizons of P2 (bamboo), P5 (teak) and P6 (paddy) suggests seasonal oxidation-reduction conditions in the studied soils (Karmakar and Rao, 1994). Alternate oxidizing and reducing conditions have been observed for the formation of $\mathrm{Fe}-\mathrm{Mn}$ concretions in alluvium derived soils of Assam (Chakravarty et al., 1982). The textural classes varied from sandy clay loam to clayey and the structure was sub angular blocky except in the surface horizon of P6 (paddy). The massive structure in the surface horizons of paddy soils is due to destruction of soil structure during puddling. Puddling damages soil structure in plough layer of paddy soils and decreases pores and voids in this layer.

Several workers have reported that the structure of the well developed soils of Assam was predominantly moderate to strong subangular blocky to massive or single grain (Chakravarty et al., 1982; Dutta et al., 2001).

\section{Physical and chemical characteristics}

A perusal of particle size distribution data of the studied soils under different land uses (Table 2) showed that B-horizons of the soils contained 1.2 times more clay content than the surface (eluvial) horizon, but no clay cutans were observed visually, confirming the absence of argillic horizons in the studied soils.

The sand and silt contents decreased with soil depth in all the profiles and clay content increased with soil depth and reached a maximum in all the soils except P7 (jackfruit) where the clay content slightly decreased after attaining the maximum. Such type of clay distribution is characteristic of moderately well developed soils (Barshad 1964). The silt content in general exhibited an irregular trend with depth which might be due to variation in weathering of parent material or in situ formation (Kumar and Naidu 2012). The significant negative correlations of clay with sand $\left(\mathrm{r}=-0.841^{* *}\right)$ and silt $\left(\mathrm{r}=-0.594^{* *}\right)$ (Table 4) suggest formation of clay from sand and silt fractions due to alteration and/or neoformation (Karmakar and Rao, 1999a) under humid sub-tropical condition.

The highest clay content within the profile and profile weighted mean in the soils of P6 under paddy land use might be attributed to lower 
topographic position of these soils and/or more pedogenic development of paddy soils. There was no abrupt changes in the ratio of silt/(silt+clay) within a profile.

The difference in silt/(silt+clay) ratios in between two adjacent horizons of a profile was less than 0.10 . This indicates uniformity of parent materials in the studied soils.

Bulk density of the studied soils showed a definite trend of increasing to a certain depth and then again it decreased except in paddy land use (P6) where irregular pattern was observed (Table 2).

The bulk density of soils is related to clay content. The significant negative correlation between bulk density and clay content ( $\mathrm{r}=$ $0.378^{*}$ ) might be due to increased in clay fraction which contributes to the development of soil micropore space, ultimately reducing the bulk density. The lower bulk density in surface layers may be due to cultivation, high organic matter and biotic activities.

The particle density was lower in the surface horizons of all the studied profiles and it increased with soil depth. Lower particle density in the surface horizons is due to high organic $\mathrm{C}$ as evident from significant negative correlation between particle density and organic $\mathrm{C}\left(\mathrm{r}=-0.873^{* *}\right)$.

The increase of particle density in the lower horizons may be attributed to higher free $\mathrm{Fe}_{2} \mathrm{O}_{3}$ and free $\mathrm{Al}_{2} \mathrm{O}_{3}$ (Table 2). This is also supported by significant positive correlations of particle density with free $\mathrm{Fe}_{2} \mathrm{O}_{3}(\mathrm{r}=0.394 *)$ and free $\mathrm{Al}_{2} \mathrm{O}_{3}(\mathrm{r}=0.539 * *)$. The sub-surface horizons, in general, had higher percentage of porosity.

This might be due to higher clay content in the sub-surface horizons as evident from significant positive correlation of porosity with clay $(\mathrm{r}=0.820 * *)$ and negative correlations with sand $(\mathrm{r}=-0.649 * *)$ and silt $\left(\mathrm{r}=-0.525^{* *}\right)$, affirming the direct contribution of clay towards the porosity of soil.

The $\mathrm{pH}(1: 2.5$ soil : water ratio) of the soils was found to be in acidic range (Table 2). The $\mathrm{pH}$ of the soil was mainly influenced by soil organic $\mathrm{C}$ and effective CEC rather than clay as evident by significant negative correlations of $\mathrm{pH}$ with organic $\mathrm{C}\left(\mathrm{r}=-0.381^{*}\right)$ and ECEC $\left(\mathrm{r}=-0.531^{* *}\right)$ and insignificant positive correlation with clay $(\mathrm{r}=210)$.

The results suggest that the organic carbon was responsible for soil acidity, and the acidity was apparently due to hydroxy-Al and organic functional groups. The electrical conductivity (EC) of the soil is proportional to its salt concentration.

The low EC (0.01 to $0.09 \mathrm{dSm}^{-1}$ ) observed indicates that the soils contain very low amount of soluble salts and there is no salinity problem in these soils. The data on soil organic carbon revealed that the soil organic carbon was higher in the surface horizon of all studied profiles and it decreased regularly with soil depth.

Higher organic carbon observed in the surface soils and was due to addition of organic matter through leaf fall, stubbles, roots etc. (Bhaskar et al., 2004).

The differences in the amount of soil organic $\mathrm{C}$ might be due to the differences in biomass addition under different land uses and also the due to differential rate of decomposition in the vicinity (Nayak et al., 2002).

The amount of free sesquioxides increased down the profile. This might be due to more soil development (Karmakar and Rao, 1999a) in the subsurface horizons. 
Table.1 Morphological characteristics of the soils

\begin{tabular}{|c|c|c|c|c|c|c|c|c|c|c|}
\hline \multirow[t]{2}{*}{ Horizon } & \multirow{2}{*}{$\begin{array}{l}\text { Depth } \\
(\mathbf{c m})\end{array}$} & \multirow{2}{*}{$\begin{array}{c}\text { Soil colour } \\
\text { (moist) }\end{array}$} & \multirow[t]{2}{*}{ Mottling } & \multirow[t]{2}{*}{ Texture } & \multirow[t]{2}{*}{ Structure } & \multicolumn{3}{|c|}{ Consistence } & \multirow{2}{*}{$\begin{array}{c}\text { Other } \\
\text { features }\end{array}$} & \multirow[t]{2}{*}{ Boundary } \\
\hline & & & & & & d & $\mathbf{m}$ & $\mathbf{w}$ & & \\
\hline \multicolumn{11}{|c|}{ P1- Acacia (Typic Dystrudepts) } \\
\hline A1 & $0-5$ & 10YR 5/4 & & scl & f $1 \mathrm{sbk}$ & $\mathrm{s}$ & vfr & ss, sp & & $\mathrm{cw}$ \\
\hline A2 & $5-20$ & 10YR 5/4 & & $\mathrm{scl}$ & f $1 \mathrm{sbk}$ & $\mathrm{s}$ & vfr & ss, sp & & gs \\
\hline Bw1 & $20-35$ & 10YR 5/4 & & $\mathrm{scl}$ & m 1 sbk & sh & fr & ss, sp & & gs \\
\hline Bw2 & $35-70$ & 10YR 6/6 & 7.5YR 7/6 & $\mathrm{scl}$ & $\mathrm{m} 2 \mathrm{sbk}$ & sh & fr & ss, sp & & gs \\
\hline Bw3 & $70-105$ & 10YR 5/6 & 7.5YR 7/6 & $\mathrm{cl}$ & m 2 sbk & $\mathrm{h}$ & fi & $\mathrm{s}, \mathrm{p}$ & & gs \\
\hline Bw4 & $105-160$ & 10YR 6/4 & 7.5YR 7/6 & $\mathrm{cl}$ & $\mathrm{m} 2 \mathrm{sbk}$ & $\mathrm{h}$ & fi & $\mathrm{s}, \mathrm{p}$ & & \\
\hline \multicolumn{10}{|c|}{ P2- Bamboo (Typic Dystrudepts) } & \\
\hline Ap & $0-15$ & 10YR 5/4 & & 1 & f $1 \mathrm{sbk}$ & $\mathrm{s}$ & fr & ss, sp & & cs \\
\hline Bw1 & $15-55$ & 10YR 5/4 & & 1 & f $1 \mathrm{sbk}$ & sh & fr & ss, sp & \multirow{4}{*}{$\begin{array}{l}\text { Few, rounded } \\
\text { to subrounded } \\
\text { Fe-Mn } \\
\text { glaebules }\end{array}$} & gs \\
\hline Bw2 & $55-85$ & 10YR 6/6 & & $\mathrm{cl}$ & m 2 sbk & sh & fr & ss, sp & & gs \\
\hline Bw3 & $85-120$ & 10YR 6/8 & 7.5YR 6/8 & $\mathrm{cl}$ & $\mathrm{m} 2 \mathrm{sbk}$ & $\mathrm{h}$ & fi & $\mathrm{s}, \mathrm{p}$ & & gs \\
\hline Bw4 & $120-165$ & 10YR 6/4 & 7.5YR 6/8 & $\mathrm{cl}$ & m 2 sbk & $\mathrm{h}$ & fi & $\mathrm{s}, \mathrm{p}$ & & \\
\hline \multicolumn{10}{|c|}{ P3- Coconut (Typic Dystrudepts) } & \\
\hline Ap & $0-15$ & 10YR 5/4 & & 1 & f $1 \mathrm{sbk}$ & $\mathrm{s}$ & fr & ss, sp & & cs \\
\hline Bw1 & $15-35$ & 10YR 5/6 & & $\mathrm{cl}$ & f $1 \mathrm{sbk}$ & sh & fr & ss, sp & & gs \\
\hline Bw2 & $35-75$ & 10YR 5/6 & 7.5YR 7/8 & $\mathrm{cl}$ & m 2 sbk & sh & fr & ss, sp & & gs \\
\hline Bw3 & $75-115$ & 10YR 7/6 & 7.5YR $7 / 8$ & $\mathrm{cl}$ & $\mathrm{m} 2 \mathrm{sbk}$ & $\mathrm{h}$ & fi & $\mathrm{s}, \mathrm{p}$ & & gs \\
\hline Bw4 & $115-180$ & 10YR 6/4 & 7.5YR 7/8 & $\mathrm{cl}$ & m 2 sbk & $\mathrm{h}$ & fi & $\mathrm{s}, \mathrm{p}$ & & \\
\hline \multicolumn{10}{|c|}{ P4- Sisoo (Typic Dystrudepts) } & \\
\hline Ap & $0-35$ & 10YR 5/4 & & $\mathrm{scl}$ & $\mathrm{f} 1 \mathrm{sbk}$ & $\mathrm{s}$ & vfr & ss, sp & & cs \\
\hline Bw1 & $35-90$ & 10YR 5/6 & 7.5YR 7/6 & $\mathrm{scl}$ & f $1 \mathrm{sbk}$ & sh & fr & ss, sp & & gs \\
\hline Bw2 & $90-125$ & 10YR 5/8 & 7.5YR 7/8 & $\mathrm{cl}$ & $\mathrm{m} 2 \mathrm{sbk}$ & sh & fi & $\mathrm{s}, \mathrm{p}$ & & gs \\
\hline Bw3 & $125-155$ & 10YR 7/6 & 7.5YR 7/8 & $\mathrm{cl}$ & $\mathrm{m} 2 \mathrm{sbk}$ & sh & fi & $\mathrm{s}, \mathrm{p}$ & & \\
\hline
\end{tabular}




\begin{tabular}{|c|c|c|c|c|c|c|c|c|c|c|}
\hline \multirow[t]{2}{*}{ Horizon } & \multirow{2}{*}{$\begin{array}{c}\text { Depth } \\
\text { (cm) }\end{array}$} & \multirow{2}{*}{$\begin{array}{l}\text { Soil colour } \\
\text { (moist) }\end{array}$} & \multirow[t]{2}{*}{ Mottling } & \multirow[t]{2}{*}{ Texture } & \multirow[t]{2}{*}{ Structure } & \multicolumn{3}{|c|}{ Consistence } & \multirow{2}{*}{$\begin{array}{c}\text { Other } \\
\text { features }\end{array}$} & \multirow[t]{2}{*}{ Boundary } \\
\hline & & & & & & $\mathrm{d}$ & $\mathrm{m}$ & $\mathrm{W}$ & & \\
\hline \multicolumn{11}{|c|}{ P5- Teak (Typic Dystrudepts) } \\
\hline Ap & $0-15$ & 10YR 5/4 & & $\mathrm{scl}$ & f $1 \mathrm{sbk}$ & $\mathrm{S}$ & $\mathrm{vfr}$ & ss, sp & \multirow{4}{*}{$\begin{array}{c}\text { Few, rounded } \\
\text { to subrounded } \\
\text { Fe-Mn } \\
\text { glaebules }\end{array}$} & cs \\
\hline Bw1 & $15-35$ & $10 Y R 5 / 6$ & & $\mathrm{scl}$ & f $1 \mathrm{sbk}$ & sh & fr & ss, sp & & gs \\
\hline Bw2 & $35-80$ & 7.5YR 5/6 & 7.5YR 7/8 & $\mathrm{cl}$ & $\mathrm{m} 2 \mathrm{sbk}$ & sh & $\mathrm{fr}$ & $\mathrm{ss}, \mathrm{sp}$ & & gs \\
\hline Bw3 & $80-160$ & 10YR 6/6 & $7.5 \mathrm{YR} 7 / 8$ & $\mathrm{cl}$ & m 2 sbk & $\mathrm{h}$ & fi & $\mathrm{s}, \mathrm{p}$ & & \\
\hline \multicolumn{11}{|c|}{ P6- Paddy (Aquic Dystrudepts) } \\
\hline Ap & $0-15$ & 10YR 4/2 & & $\mathrm{cl}$ & $\mathrm{m}$ & sh & fr & ss, sp & \multirow{5}{*}{$\begin{array}{c}\text { Few, rounded } \\
\text { to subrounded } \\
\text { Fe-Mn } \\
\text { glaebules }\end{array}$} & cs \\
\hline Bw1 & $15-35$ & 10YR 5/4 & & $\mathrm{cl}$ & f $1 \mathrm{sbk}$ & sh & fr & ss, sp & & gs \\
\hline Bw2 & $35-60$ & $10 Y R$ 6/8 & 7.5 YR $6 / 8$ & $\mathrm{c}$ & m $3 \mathrm{sbk}$ & $\mathrm{h}$ & fi & $\mathrm{s}, \mathrm{p}$ & & gs \\
\hline Bw3 & $60-105$ & 10YR 6/4 & $7.5 \mathrm{YR} 6 / 8$ & $\mathrm{cl}$ & m $2 \mathrm{sbk}$ & $\mathrm{h}$ & fi & $\mathrm{s}, \mathrm{p}$ & & gs \\
\hline Bw4 & $105-160$ & $10 Y R 6 / 8$ & $7.5 \mathrm{YR} 6 / 8$ & $\mathrm{cl}$ & m $2 \mathrm{sbk}$ & $\mathrm{h}$ & fi & $\mathrm{s}, \mathrm{p}$ & & \\
\hline \multicolumn{11}{|c|}{ P7- Jackfruit (Typic Dystrudepts) } \\
\hline Ap & $0-15$ & 10YR 5/4 & & scl & f $1 \mathrm{sbk}$ & $\mathrm{S}$ & $\mathrm{vfr}$ & ss, sp & & cs \\
\hline Bw1 & $15-25$ & $10 Y R 5 / 4$ & & $\mathrm{scl}$ & f $1 \mathrm{sbk}$ & sh & vfr & $\mathrm{ss}, \mathrm{sp}$ & & gs \\
\hline Bw2 & $25-55$ & 10YR 5/6 & 7.5YR 7/8 & $\mathrm{cl}$ & $\mathrm{m} 1 \mathrm{sbk}$ & sh & $\mathrm{fr}$ & $\mathrm{ss}, \mathrm{sp}$ & & gs \\
\hline Bw3 & $55-70$ & 10YR 6/8 & 7.5YR 7/8 & $\mathrm{cl}$ & $\mathrm{m} 2 \mathrm{sbk}$ & sh & fr & ss, sp & & gs \\
\hline Bw4 & $70-105$ & 10YR 6/8 & 7.5YR 7/8 & $\mathrm{cl}$ & m $2 \mathrm{sbk}$ & $\mathrm{h}$ & fi & $\mathrm{s}, \mathrm{p}$ & & gs \\
\hline Bw5 & $105-165$ & 10YR 6/8 & $7.5 \mathrm{YR} 7 / 8$ & $\mathrm{cl}$ & m $2 \mathrm{sbk}$ & $\mathrm{h}$ & fi & $\mathrm{s}, \mathrm{p}$ & & \\
\hline
\end{tabular}

Boundary: cs - clear smooth; gs - gradual smooth

Texture: ls - loamy sand; sl - sandy loam; scl - sandy clay loam; cl - clay loam; sil - silt loam; sicl - silty clay loam; 1 - loam; sic - silty clay; c - clay.

Structure: $\mathrm{f}$ - fine, $\mathrm{m}$ - medium, c - coarse; 1 - weak, 2 - moderate, 3 - strong; sg - single grained, $\mathrm{m}$ - massive, sbk - subangular blocky, abk - angular blocky.

Consistency: d(dry): 1 - loose, s - soft, sh - slightly hard, h - hard, vh - very hard; m(moist): vfr - very friable, fr - friable, fi - firm; w(wet): ss - slightly sticky, s - sticky; sp -

slightly plastic, $\mathrm{p}$ - plastic.

Mottles: Abundance: $\mathrm{f}$ - few, $\mathrm{m}$ - common; Size: 1 - fine, 2 - medium; Contrast: $\mathrm{f}$ - faint, $\mathrm{d}$ - distinct, $\mathrm{p}$ - prominent 
Table.2 Physical and chemical properties of the studied soils

\begin{tabular}{|c|c|c|c|c|c|c|c|c|c|c|c|c|c|}
\hline \multirow[t]{3}{*}{ Horizon } & \multirow{3}{*}{$\begin{array}{l}\text { Depth } \\
\text { (cm) }\end{array}$} & \multicolumn{3}{|c|}{ Particle size distribution (mm) } & \multirow{3}{*}{$\begin{array}{c}\text { Silt / } \\
\text { Silt+clay }\end{array}$} & \multirow{3}{*}{$\begin{array}{c}\text { Bulk } \\
\text { density } \\
\left(\mathrm{Mg} \mathrm{m}^{-3}\right)\end{array}$} & \multirow{3}{*}{$\begin{array}{l}\text { Particle } \\
\text { density } \\
\left(\mathrm{Mg} \mathrm{m}^{-3}\right)\end{array}$} & \multirow{3}{*}{$\begin{array}{c}\text { Porosity } \\
(\%)\end{array}$} & \multirow[t]{3}{*}{ pH } & \multirow[t]{3}{*}{ E.C } & \multirow{3}{*}{$\begin{array}{c}\text { Organic C } \\
\left(\mathrm{g} \mathrm{kg}^{-1}\right)\end{array}$} & \multirow{3}{*}{$\begin{array}{c}\text { Free } \\
\mathrm{Fe}_{2} \mathrm{O}_{3} \\
(\%)\end{array}$} & \multirow{3}{*}{$\begin{array}{c}\text { Free } \\
\mathrm{Al}_{2} \mathrm{O}_{3} \\
(\%)\end{array}$} \\
\hline & & $\begin{array}{l}\text { Sand } \\
(2-0.05)\end{array}$ & $\begin{array}{c}\text { Silt } \\
(0.05-0.002)\end{array}$ & $\begin{array}{c}\text { Clay } \\
(<0.002)\end{array}$ & & & & & & & & & \\
\hline & & \multicolumn{3}{|c|}{$\stackrel{\%}{\longrightarrow} \longrightarrow$} & & & & & & & & & \\
\hline \multicolumn{14}{|c|}{ P1: Acacia (Typic Dystrudepts) } \\
\hline A1 & $0-5$ & 50.1 & 27.8 & 22.1 & 0.56 & 1.36 & 2.42 & 43.80 & 4.9 & 0.02 & 7.6 & 1.80 & 0.58 \\
\hline A2 & $5-20$ & 49.6 & 24.6 & 25.8 & 0.49 & 1.38 & 2.48 & 44.35 & 5.1 & 0.04 & 4.9 & 1.86 & 0.57 \\
\hline Bw1 & $20-35$ & 46.6 & 25.6 & 27.8 & 0.48 & 1.46 & 2.53 & 42.29 & 5.1 & 0.06 & 3.1 & 1.88 & 0.58 \\
\hline Bw2 & $35-70$ & 46.1 & 24.1 & 29.8 & 0.45 & 1.38 & 2.56 & 46.09 & 5.3 & 0.06 & 1.3 & 1.84 & 0.60 \\
\hline Bw3 & $70-105$ & 43.7 & 23.7 & 32.6 & 0.42 & 1.37 & 2.55 & 46.27 & 5.4 & 0.06 & 1.1 & 1.90 & 0.60 \\
\hline Bw4 & $105-160$ & 40.4 & 22.7 & 36.9 & 0.38 & 1.33 & 2.61 & 49.04 & 5.6 & 0.07 & 0.7 & 1.90 & 0.60 \\
\hline \multicolumn{2}{|c|}{ Wt. mean } & 44.1 & 23.8 & 32.1 & & 1.37 & 2.56 & 46.56 & 5.3 & 0.06 & 1.7 & 1.88 & 0.59 \\
\hline \multicolumn{14}{|c|}{ P2: Bamboo (Typic Dystrudepts) } \\
\hline Ap & $0-15$ & 50.6 & 28.1 & 21.3 & 0.57 & 1.38 & 2.40 & 42.58 & 5.3 & 0.04 & 6.7 & 1.79 & 0.57 \\
\hline Bw1 & $15-55$ & 44.7 & 30.5 & 24.8 & 0.55 & 1.42 & 2.49 & 42.97 & 5.5 & 0.06 & 2.6 & 1.84 & 0.68 \\
\hline Bw2 & $55-85$ & 45.0 & 26.1 & 28.9 & 0.47 & 1.38 & 2.51 & 45.02 & 5.6 & 0.06 & 1.7 & 1.90 & 0.75 \\
\hline Bw3 & $85-120$ & 43.2 & 24.2 & 32.6 & 0.43 & 1.41 & 2.54 & 44.49 & 5.7 & 0.07 & 1.3 & 1.96 & 0.89 \\
\hline Bw4 & $120-165$ & 42.0 & 21.1 & 36.9 & 0.36 & 1.41 & 2.60 & 45.77 & 5.8 & 0.09 & 0.7 & 1.90 & 1.08 \\
\hline \multicolumn{2}{|c|}{ Wt. mean } & 44.2 & 25.6 & 30.2 & & 1.40 & 2.53 & 44.39 & 5.6 & 0.07 & 2.1 & 1.89 & 0.84 \\
\hline \multicolumn{14}{|c|}{ P3: Coconut (Typic Dystrudepts) } \\
\hline Ap & $0-15$ & 45.1 & 28.8 & 26.1 & 0.52 & 1.35 & 2.42 & 44.15 & 5.1 & 0.03 & 5.8 & 1.54 & 0.61 \\
\hline Bw1 & $15-35$ & 39.5 & 31.6 & 28.9 & 0.52 & 1.39 & 2.47 & 43.72 & 5.3 & 0.05 & 4.0 & 1.58 & 0.68 \\
\hline Bw2 & $35-75$ & 42.8 & 25.6 & 31.6 & 0.45 & 1.35 & 2.56 & 47.27 & 5.3 & 0.05 & 2.6 & 1.64 & 0.76 \\
\hline Bw3 & $75-115$ & 39.4 & 23.8 & 36.8 & 0.39 & 1.37 & 2.58 & 46.90 & 5.4 & 0.06 & 2.1 & 1.70 & 0.82 \\
\hline Bw4 & $115-180$ & 38.3 & 22.9 & 38.8 & 0.37 & 1.35 & 2.57 & 47.47 & 5.2 & 0.04 & 1.0 & 1.80 & 0.89 \\
\hline \multicolumn{2}{|c|}{ Wt. mean } & 40.2 & 25.2 & 34.6 & & 1.36 & 2.55 & 46.61 & 5.2 & 0.05 & 2.3 & 1.70 & 0.80 \\
\hline \multicolumn{14}{|c|}{ P4: Sisoo (Typic Dystrudepts) } \\
\hline Ap & $0-35$ & 49.2 & 24.4 & 26.3 & 0.48 & 1.37 & 2.48 & 44.68 & 5.1 & 0.03 & 6.5 & 1.67 & 0.66 \\
\hline Bw1 & $35-90$ & 45.8 & 24.4 & 29.8 & 0.45 & 1.41 & 2.57 & 45.14 & 5.2 & 0.04 & 3.3 & 1.74 & 0.74 \\
\hline Bw2 & $90-125$ & 41.3 & 24.1 & 34.6 & 0.41 & 1.37 & 2.57 & 46.69 & 5.2 & 0.04 & 3.0 & 1.81 & 0.88 \\
\hline Bw3 & $125-155$ & 41.3 & 20.9 & 37.8 & 0.36 & 1.36 & 2.61 & 47.89 & 5.4 & 0.06 & 0.3 & 1.84 & 1.02 \\
\hline \multicolumn{2}{|c|}{ Wt. mean } & 44.7 & 23.7 & 31.7 & & 1.38 & 2.56 & 45.92 & 5.2 & 0.04 & 3.4 & 1.76 & 0.81 \\
\hline
\end{tabular}




\begin{tabular}{|c|c|c|c|c|c|c|c|c|c|c|c|c|c|}
\hline \multirow[t]{3}{*}{ Horizon } & \multirow{3}{*}{$\begin{array}{l}\text { Depth } \\
\text { (cm) }\end{array}$} & \multicolumn{3}{|c|}{ Particle size distribution (mm) } & \multirow{3}{*}{$\begin{array}{c}\text { Silt / } \\
\text { Silt+clay }\end{array}$} & \multirow{3}{*}{$\begin{array}{c}\text { Bulk } \\
\text { density } \\
(\mathrm{Mg} \\
\left.\mathrm{m}^{-3}\right)\end{array}$} & \multirow{3}{*}{$\begin{array}{l}\text { Particledensity } \\
\quad\left(\mathrm{Mg} \mathrm{m}^{-3}\right)\end{array}$} & \multirow{3}{*}{$\begin{array}{c}\text { Porosity } \\
(\%)\end{array}$} & \multirow[t]{3}{*}{ pH } & \multirow[t]{3}{*}{ E.C } & \multirow{3}{*}{$\begin{array}{c}\text { Organic } \\
\mathrm{C} \\
\left(\mathrm{g} \mathrm{kg}^{-1}\right)\end{array}$} & \multirow{3}{*}{$\begin{array}{c}\text { Free } \\
\mathrm{Fe}_{2} \mathrm{O}_{3} \\
(\%)\end{array}$} & \multirow{3}{*}{$\begin{array}{c}\text { Free } \\
\mathrm{Al}_{2} \mathrm{O}_{3} \\
(\%)\end{array}$} \\
\hline & & $\begin{array}{c}\text { Sand } \\
(2- \\
0.05)\end{array}$ & $\begin{array}{c}\text { Silt } \\
(0.05- \\
\text { 0.002) }\end{array}$ & $\begin{array}{c}\text { Clay } \\
(<0.002)\end{array}$ & & & & & & & & & \\
\hline & & \multicolumn{3}{|c|}{$\stackrel{\text { कै }}{\longleftarrow}$} & & & & & & & & & \\
\hline \multicolumn{14}{|c|}{ P5: Teak (Typic Dystrudepts) } \\
\hline Ap & $0-15$ & 50.9 & 23.1 & 26.0 & 0.47 & 1.37 & 2.48 & 44.76 & 5.1 & 0.04 & 5.1 & 1.64 & 0.58 \\
\hline Bw1 & $15-35$ & 48.8 & 22.2 & 29.0 & 0.43 & 1.38 & 2.56 & 46.09 & 5.3 & 0.05 & 3.8 & 1.67 & 0.78 \\
\hline Bw2 & $35-80$ & 44.8 & 22.8 & 32.4 & 0.41 & 1.47 & 2.57 & 42.80 & 5.5 & 0.05 & 2.6 & 1.82 & 0.86 \\
\hline Bw3 & $80-160$ & 42.8 & 20.8 & 36.4 & 0.36 & 1.37 & 2.60 & 47.31 & 5.6 & 0.07 & 0.3 & 1.87 & 0.89 \\
\hline \multicolumn{2}{|c|}{ Wt. mean } & 44.9 & 21.8 & 33.4 & & 1.40 & 2.58 & 45.65 & 5.5 & 0.06 & 1.8 & 1.81 & 0.84 \\
\hline \multicolumn{14}{|c|}{ P6: Paddy (Aquic Dystrudepts) } \\
\hline Ap & $0-15$ & 38.1 & 28.1 & 33.8 & 0.45 & 1.33 & 2.48 & 46.17 & 5.4 & 0.06 & 7.0 & 1.70 & 0.52 \\
\hline Bw1 & $15-35$ & 36.5 & 26.7 & 36.8 & 0.42 & 1.35 & 2.51 & 46.22 & 5.1 & 0.04 & 2.3 & 1.64 & 0.64 \\
\hline Bw2 & $35-60$ & 35.5 & 24.4 & 40.1 & 0.38 & 1.31 & 2.56 & 48.83 & 5.0 & 0.03 & 1.3 & 1.56 & 0.86 \\
\hline Bw3 & $60-105$ & 40.8 & 20.8 & 38.4 & 0.35 & 1.38 & 2.56 & 46.09 & 4.9 & 0.02 & 1.3 & 1.57 & 0.78 \\
\hline Bw4 & $\begin{array}{l}105- \\
160\end{array}$ & 39.7 & 23.4 & 36.9 & 0.39 & 1.37 & 2.59 & 47.10 & 4.7 & 0.01 & 0.3 & 1.85 & 0.86 \\
\hline \multicolumn{2}{|c|}{ Wt. mean } & 38.8 & 23.7 & 37.5 & & 1.36 & 2.56 & 46.89 & 4.9 & 0.02 & 1.6 & 1.69 & 0.78 \\
\hline \multicolumn{14}{|c|}{ P7: Jackfruit (Typic Dystrudepts) } \\
\hline Ap & $0-15$ & 49.5 & 24.1 & 26.3 & 0.47 & 1.38 & 2.45 & 43.67 & 4.9 & 0.04 & 6.4 & 1.61 & 0.52 \\
\hline Bw1 & $15-25$ & 47.4 & 22.8 & 29.8 & 0.43 & 1.41 & 2.49 & 43.37 & 5.4 & 0.05 & 4.6 & 1.70 & 0.68 \\
\hline Bw2 & $25-55$ & 44.5 & 24.1 & 31.4 & 0.43 & 1.36 & 2.51 & 45.82 & 5.4 & 0.06 & 2.6 & 1.74 & 0.78 \\
\hline Bw3 & $55-70$ & 40.8 & 24.6 & 34.6 & 0.42 & 1.37 & 2.56 & 46.48 & 5.3 & 0.06 & 0.9 & 1.80 & 1.76 \\
\hline Bw4 & $70-105$ & 43.1 & 22.3 & 38.7 & 0.37 & 1.33 & 2.59 & 48.65 & 5.5 & 0.07 & 0.8 & 1.80 & 0.89 \\
\hline Bw5 & $\begin{array}{l}105- \\
165\end{array}$ & 39.6 & 20.6 & 39.8 & 0.34 & 1.34 & 2.60 & 48.46 & 5.5 & 0.07 & 0.3 & 1.89 & 1.02 \\
\hline \multicolumn{2}{|c|}{ Wt. mean } & 42.7 & 22.4 & 35.7 & & 1.35 & 2.56 & 47.10 & 5.4 & 0.06 & 1.7 & 1.80 & 0.95 \\
\hline
\end{tabular}


Table.3 Exchangeable cations, exchangeable acidity, cation exchange capacity (CEC), effective cation exchange capacity (ECEC) and base saturation of the studied soils

\begin{tabular}{|c|c|c|c|c|c|c|c|c|c|c|c|c|}
\hline \multirow[t]{2}{*}{ Horizon } & Depth & Exch. $\mathrm{Ca}^{2+}$ & $\begin{array}{l}\text { Exch. } \\
\mathrm{Mg}^{2+}\end{array}$ & Exch. $\mathbf{K}^{+}$ & Exch. $\mathbf{N a}^{+}$ & \multirow[t]{2}{*}{$\mathrm{Ca} / \mathrm{Mg}$} & Exch. $\mathbf{H}^{+}$ & $\begin{array}{l}\text { Exch. } \\
\mathbf{A l}^{3+}\end{array}$ & $\begin{array}{l}\text { Exch. } \\
\text { acidity }\end{array}$ & CEC & ECEC & \multirow{2}{*}{$\begin{array}{c}\text { Base } \\
\text { Saturation } \\
(\%)\end{array}$} \\
\hline & (cm) & \multicolumn{4}{|c|}{4} & & \multicolumn{3}{|c|}{$\longleftarrow$} & \multicolumn{2}{|c|}{$\operatorname{cmol}\left(p_{+}\right) \mathrm{kg}^{-1}$} & \\
\hline \multicolumn{13}{|c|}{ P1: Acacia (Typic Dystrudepts) } \\
\hline A1 & $0-5$ & 2.75 & 1.75 & 0.37 & 0.15 & 1.57 & 0.10 & 0.55 & 0.65 & 9.10 & 5.32 & 52.4 \\
\hline A2 & $5-20$ & 2.20 & 1.50 & 0.27 & 0.08 & 1.46 & 0.20 & 0.45 & 0.65 & 7.11 & 4.36 & 55.0 \\
\hline Bw1 & $20-35$ & 2.40 & 1.15 & 0.25 & 0.10 & 2.09 & 0.15 & 0.55 & 0.70 & 6.96 & 4.27 & 53.4 \\
\hline Bw2 & $35-70$ & 2.20 & 1.25 & 0.10 & 0.10 & 1.76 & 0.15 & 0.45 & 0.60 & 6.70 & 4.06 & 53.9 \\
\hline Bw3 & $70-105$ & 1.80 & 1.00 & 0.15 & 0.07 & 1.80 & 0.10 & 0.35 & 0.45 & 5.90 & 3.24 & 49.0 \\
\hline Bw4 & $105-160$ & 1.80 & 1.20 & 0.11 & 0.08 & 1.50 & 0.10 & 0.25 & 0.35 & 7.40 & 3.34 & 41.8 \\
\hline \multicolumn{2}{|c|}{ Wt. mean } & 2.01 & 1.21 & 0.15 & 0.09 & & 0.13 & 0.37 & 0.50 & 6.90 & 3.72 & 48.7 \\
\hline \multicolumn{13}{|c|}{ P2: Bamboo (Typic Dystrudepts) } \\
\hline Ap & $0-15$ & 2.80 & 1.42 & 0.23 & 0.19 & 2.03 & 0.32 & 0.18 & 0.50 & 7.80 & 4.59 & 57.2 \\
\hline Bw1 & $15-55$ & 2.60 & 1.15 & 0.08 & 0.10 & 2.26 & 0.25 & 0.20 & 0.45 & 6.98 & 4.15 & 56.6 \\
\hline Bw2 & $55-85$ & 2.40 & 1.00 & 0.08 & 0.07 & 2.40 & 0.15 & 0.35 & 0.50 & 6.74 & 3.92 & 53.0 \\
\hline Bw3 & $85-120$ & 2.10 & 0.75 & 0.11 & 0.04 & 2.80 & 0.10 & 0.15 & 0.25 & 5.18 & 3.10 & 56.9 \\
\hline Bw4 & $120-165$ & 2.10 & 0.75 & 0.11 & 0.04 & 2.80 & 0.10 & 0.10 & 0.20 & 7.10 & 3.05 & 41.5 \\
\hline \multicolumn{2}{|c|}{ Wt. mean } & 2.34 & 0.95 & 0.11 & 0.07 & & 0.17 & 0.19 & 0.35 & 6.66 & 3.63 & 52.0 \\
\hline \multicolumn{13}{|c|}{ P3: Coconut (Typic Dystrudepts) } \\
\hline Ap & $0-15$ & 2.45 & 1.83 & 0.11 & 0.09 & 1.34 & 0.20 & 0.33 & 0.53 & 7.97 & 4.83 & 56.4 \\
\hline Bw1 & $15-35$ & 1.70 & 1.25 & 0.10 & 0.07 & 1.36 & 0.15 & 0.30 & 0.45 & 6.01 & 3.40 & 51.6 \\
\hline Bw2 & $35-75$ & 1.80 & 1.25 & 0.06 & 0.06 & 1.44 & 0.20 & 0.25 & 0.45 & 6.77 & 3.76 & 51.8 \\
\hline Bw3 & $75-115$ & 1.80 & 0.75 & 0.04 & 0.03 & 2.40 & 0.10 & 0.35 & 0.45 & 6.18 & 2.97 & 42.4 \\
\hline Bw4 & $115-180$ & 2.00 & 1.00 & 0.08 & 0.04 & 2.00 & 0.10 & 0.45 & 0.55 & 7.00 & 3.50 & 43.6 \\
\hline \multicolumn{2}{|c|}{ Wt. mean } & 1.92 & 1.10 & 0.07 & 0.05 & & 0.14 & 0.36 & 0.49 & 6.74 & 3.54 & 47.1 \\
\hline \multicolumn{13}{|c|}{ P4: Sisoo (Typic Dystrudepts) } \\
\hline Ap & $0-35$ & 1.83 & 1.46 & 0.25 & 0.15 & 1.27 & 0.14 & 0.32 & 0.46 & 7.90 & 3.90 & 45.0 \\
\hline Bw1 & $35-90$ & 1.70 & 1.50 & 0.25 & 0.10 & 1.13 & 0.10 & 0.20 & 0.30 & 7.40 & 3.62 & 46.2 \\
\hline Bw2 & $90-125$ & 1.80 & 1.50 & 0.18 & 0.12 & 1.20 & 0.05 & 0.15 & 0.20 & 8.11 & 3.63 & 42.9 \\
\hline Bw3 & $125-155$ & 2.20 & 1.00 & 0.11 & 0.10 & 2.20 & 0.05 & 0.10 & 0.15 & 8.11 & 3.42 & 40.9 \\
\hline \multicolumn{2}{|c|}{ Wt. mean } & 1.85 & 1.39 & 0.21 & 0.12 & & 0.09 & 0.20 & 0.29 & 7.81 & 3.64 & 44.2 \\
\hline
\end{tabular}




\begin{tabular}{|c|c|c|c|c|c|c|c|c|c|c|c|c|}
\hline \multirow[t]{2}{*}{ Horizon } & Depth & $\begin{array}{c}\text { Exch. } \\
\mathrm{Ca}^{2+}\end{array}$ & $\begin{array}{l}\text { Exch. } \\
\mathrm{Mg}^{2+}\end{array}$ & $\begin{array}{c}\text { Exch. } \\
\mathbf{K}^{+}\end{array}$ & $\begin{array}{c}\text { Exch. } \\
\mathrm{Na}^{+}\end{array}$ & \multirow[t]{2}{*}{$\mathrm{Ca} / \mathrm{Mg}$} & $\underset{\mathbf{H}^{+}}{\text {Exch. }}$ & $\underset{\mathbf{A l}^{3+}}{\text { Exch. }}$ & $\begin{array}{c}\text { Exch. } \\
\text { acidity }\end{array}$ & CEC & ECEC & \multirow{2}{*}{$\begin{array}{c}\text { Base } \\
\text { Saturation } \\
(\%)\end{array}$} \\
\hline & $(\mathbf{c m})$ & \multicolumn{2}{|c|}{$\longleftarrow$} & \multicolumn{2}{|c|}{$\operatorname{cmol}(p+) \mathrm{kg}^{-1} \longrightarrow$} & & \multicolumn{2}{|c|}{$\longleftarrow$} & \multicolumn{3}{|c|}{ cmel $(\mathrm{p}+) \mathrm{kg}^{-1} \longrightarrow$} & \\
\hline \multicolumn{13}{|c|}{ P5: Teak (Typic Dystrudepts) } \\
\hline Ap & $0-15$ & 2.10 & 1.60 & 0.18 & 0.14 & 1.31 & 0.35 & 0.40 & 0.75 & 8.98 & 4.36 & 44.1 \\
\hline Bw1 & $15-35$ & 1.60 & 1.20 & 0.11 & 0.12 & 1.33 & 0.20 & 0.35 & 0.55 & 7.11 & 3.38 & 42.6 \\
\hline Bw2 & $35-80$ & 1.85 & 1.00 & 0.13 & 0.12 & 1.85 & 0.15 & 0.20 & 0.35 & 6.71 & 3.21 & 44.9 \\
\hline Bw3 & $80-160$ & 1.80 & 1.25 & 0.10 & 0.08 & 1.44 & 0.10 & 0.10 & 0.20 & 7.54 & 3.25 & 41.8 \\
\hline \multicolumn{2}{|c|}{ Wt. mean } & 2.73 & 1.17 & 0.12 & 0.10 & & 0.15 & 0.19 & 0.34 & 7.39 & 4.23 & 54.8 \\
\hline \multicolumn{13}{|c|}{ P6: Paddy (Aquic Dystrudepts) } \\
\hline Ap & $0-15$ & 1.80 & 1.67 & 0.30 & 0.15 & 1.09 & 0.33 & 0.45 & 0.78 & 8.4 & 4.3 & 45.8 \\
\hline Bw1 & $15-35$ & 2.10 & 1.25 & 0.13 & 0.12 & 1.68 & 0.30 & 0.25 & 0.55 & 7.10 & 3.81 & 50.1 \\
\hline Bw2 & $35-60$ & 2.20 & 1.30 & 0.10 & 0.12 & 1.69 & 0.10 & 0.20 & 0.30 & 8.10 & 3.86 & 45.2 \\
\hline Bw3 & $60-105$ & 2.00 & 1.25 & 0.09 & 0.08 & 1.60 & 0.05 & 0.15 & 0.20 & 7.18 & 3.52 & 46.9 \\
\hline Bw4 & $105-160$ & 2.20 & 1.25 & 0.09 & 0.05 & 1.76 & 0.05 & 0.15 & 0.20 & 8.10 & 3.71 & 44.0 \\
\hline \multicolumn{2}{|c|}{ Wt. mean } & 2.09 & 1.30 & 0.12 & 0.09 & & 0.12 & 0.20 & 0.31 & 7.75 & 3.75 & 45.9 \\
\hline \multicolumn{13}{|c|}{ P7: Jackfruit (Typic Dystrudepts) } \\
\hline Ap & $0-15$ & 2.68 & 1.92 & 0.30 & 0.13 & 1.41 & 0.17 & 0.23 & 0.40 & 10.30 & 5.10 & 47.2 \\
\hline Bw1 & $15-25$ & 2.50 & 1.20 & 0.23 & 0.09 & 2.08 & 0.15 & 0.25 & 0.40 & 9.10 & 4.16 & 43.0 \\
\hline Bw2 & $25-55$ & 1.55 & 1.25 & 0.10 & 0.07 & 1.24 & 0.10 & 0.25 & 0.35 & 8.10 & 3.16 & 35.9 \\
\hline Bw3 & $55-70$ & 1.55 & 1.50 & 0.09 & 0.07 & 1.03 & 0.10 & 0.20 & 0.30 & 7.90 & 3.40 & 40.5 \\
\hline Bw4 & $70-105$ & 2.20 & 1.20 & 0.11 & 0.09 & 1.83 & 0.05 & 0.10 & 0.15 & 8.40 & 3.60 & 41.7 \\
\hline Bw5 & $105-165$ & 2.60 & 1.20 & 0.12 & 0.03 & 2.17 & 0.05 & 0.10 & 0.15 & 8.40 & 3.94 & 45.7 \\
\hline \multicolumn{2}{|c|}{ Wt. mean } & 2.23 & 1.30 & 0.14 & 0.07 & 0.07 & 0.08 & 0.16 & 0.24 & 8.52 & 3.80 & 42.6 \\
\hline
\end{tabular}


Table.4 Correlation coefficients (r) among selected soil properties

\begin{tabular}{|c|c|c|c|c|c|c|c|c|c|c|c|}
\hline & Sand & Silt & Clay & $\begin{array}{c}\text { Bulk } \\
\text { density }\end{array}$ & $\begin{array}{l}\text { Particle } \\
\text { density }\end{array}$ & Porosity & pH & $\begin{array}{c}\text { Organic } \\
\text { C }\end{array}$ & $\mathrm{Fe}_{2} \mathrm{O}_{3}$ & $\mathrm{Al}_{2} \mathrm{O}_{3}$ & ECEC \\
\hline Silt & 0.084 & 1.000 & & & & & & & & & \\
\hline Clay & $-0.841 * *$ & $-0.594 * *$ & 1.000 & & & & & & & & \\
\hline Bulk density & $0.402 *$ & 0.055 & $-0.378 *$ & 1.000 & & & & & & & \\
\hline Particle density & $-0.547 * *$ & $-0.718 * *$ & $0.823 * *$ & -0.062 & 1.000 & & & & & & \\
\hline Porosity & $-0.649 * *$ & $-0.525 * *$ & $0.820 * *$ & -0.738 & 0.719 & 1.000 & & & & & \\
\hline pH & -0.132 & -0.165 & 0.210 & 0.151 & 0.350 & 0.128 & 1.000 & & & & \\
\hline Organic C & $0.606 * *$ & 0.545 & $-0.782 * *$ & 0.061 & $-0.873 * *$ & -0.636 & $-0.381 * *$ & 1.000 & & & \\
\hline Free $\mathrm{Fe}_{2} \mathrm{O}_{3}$ & 0.043 & -0.275 & 0.114 & 0.276 & $0.394 *$ & 0.071 & $0.526 * *$ & -0.445 & 1.000 & & \\
\hline Free $\mathrm{Al}_{2} \mathrm{O}_{3}$ & -0.398 & -0.373 & 0.519 & -0.032 & $0.539 * *$ & 0.388 & 0.261 & -0.573 & 0.230 & 1.000 & \\
\hline Exch. H+ & 0.321 & 0.531 & $-0.551 * *$ & 0.049 & -0.631 & -0.460 & -0.065 & $0.601 * *$ & -0.320 & -0.486 & \\
\hline Exch. $\mathbf{A l}^{3+}$ & 0.327 & 0.385 & $-0.485^{* *}$ & 0.052 & -0.452 & -0.341 & -0.309 & $0.496 * *$ & -0.093 & -0.500 & \\
\hline Exch. acidity & 0.387 & 0.527 & $-0.609 * *$ & 0.061 & -0.622 & -0.462 & -0.255 & $0.640 * *$ & -0.216 & -0.589 & \\
\hline CEC & 0.265 & -0.123 & -0.130 & -0.315 & -0.284 & 0.023 & -0.411 & $0.415 *$ & -0.296 & -0.064 & \\
\hline ECEC & 0.516 & 0.451 & -0.649 & -0.085 & -0.758 & -0.457 & -0.531 & $0.732 * *$ & -0.268 & -0.512 & 1.000 \\
\hline Base saturation & 0.299 & 0.642 & $-0.586^{*}$ & 0.250 & -0.565 & -0.555 & -0.121 & $0.344 *$ & 0.036 & -0.463 & 0.510 \\
\hline
\end{tabular}

' $r$ ' values for $33 \mathrm{df}=0.345$ at $\mathrm{P}_{0.05}, 0.443$ at $\mathrm{P}_{0.01}$ 
The positive correlations of clay with free $\mathrm{Fe}_{2} \mathrm{O}_{3} \quad(\mathrm{r}=0.114)$ and $\mathrm{Al}_{2} \mathrm{O}_{3} \quad\left(\mathrm{r}=0.519^{* *}\right)$ indicate co-migration and/or in situ formation of clay and free sesquioxides.

\section{Exchangeable cations, CEC and base saturation}

Exchange complex was mostly dominated by $\mathrm{Ca}^{2+}$ followed by $\mathrm{Mg}^{2+}, \mathrm{Na}^{+}$and $\mathrm{K}^{+}$(Table 3). The dominance of exchangeable $\mathrm{Ca}^{2+}$ in these soils is in general agreement with the findings of earlier workers on soils of Assam (Chakravarty et al., 1978; Dey and Sehgal 1997). The $\mathrm{Ca}^{2+} / \mathrm{Mg}^{2+}$ ratio indicates considerable recycling of $\mathrm{Ca}^{2+}$ as compared to $\mathrm{Mg}^{2+}$ during the process of soil formation (Gangopadhyay et al., 2001). Low amounts of exchangeable $\mathrm{K}^{+}$and $\mathrm{Na}^{+}$as compared to $\mathrm{Ca}^{2+}$ and $\mathrm{Mg}^{2+}$ might be attributed to preferential leaching of $\mathrm{K}^{+}$and $\mathrm{Na}^{+}$over divalent cations. The exchangeable $\mathrm{Al}^{3+}$ was dominant over exchangeable $\mathrm{H}^{+}$ion in all the studied land uses. The exchangeable $\mathrm{Al}^{3+}$ was significantly and positively correlated with organic $\mathrm{C}$ ( $\mathrm{r}=$ $\left.0.496^{* *}\right)$ and negatively correlated with $\mathrm{pH}$ $(\mathrm{r}=-0.309)$ (Table 4). The significant positive correlations of organic $\mathrm{C}$ with exchangeable $\mathrm{H}^{+} \quad\left(\mathrm{r}=0.601^{* *}\right)$, exchangeable $\mathrm{Al}^{3+} \quad(\mathrm{r}=$ $\left.0.496^{* *}\right)$ and exchange acidity $\left(\mathrm{r}=0.640^{* *}\right)$ suggest more contribution of soil organic matter towards soil acidity components.

The cation exchange capacity (CEC) of the soils varied narrowly within a profile with an apparent irregular trend with soil depth (Table $3)$. The CEC of the soil, in general was low $[<$ $\left.15 \mathrm{cmol}\left(\mathrm{p}^{+}\right) \mathrm{kg}^{-1}\right]$. Low CEC in the studied soils may be due to presence of low activity clay (kaolinite) (Bhattacharyya et al., 1994) as the CEC of soils is immensely affected by the mineralogy of the soil. The CEC of the studied soils is a function of organic matter rather than the clay content. This is corroborated by significant positive correlation between CEC and soil organic $\mathrm{C}\left(\mathrm{r}=0.415^{* *}\right)$. The effective cation exchange capacity (ECEC) of the soils (CEC of soil by $1 \mathrm{~N}$ ammonium acetate at $\mathrm{pH}$ $7.0+1 \mathrm{~N} \mathrm{KCl}$ extractable aluminium) was found to be low. On the basis of profile weighted mean, the base saturation was found to be in the sequence of P5 (teak) $(54.8 \%)>$ P2 (bamboo) (52.0\%) > P1 (acacia) $(48.7 \%)>$ P3 (coconut) $(47.1 \%)>$ P6 (paddy) $(45.9 \%)>$ P4 (sisoo) $(44.2 \%)>$ P7 (jackfruit) $(42.6 \%)$. Highest base saturation under teak vegetation was due to high amount of bases and low soil CEC (Patil and Dasog 1999). The variation observed in base saturation indicates the degree of leaching of bases, which is used as one of the diagnostic criteria for classifying the soil orders.

\section{Soil classification}

The soils of the study area have ochric epipedons, cambic subsurface horizons and udic soil moisture regime. So these soils were classified as Inceptisols order and Udepts suborder. The base saturation (by $1 \mathrm{~N}$ $\mathrm{NH}_{4} \mathrm{OAc}$ ) of these soils was less than 60 percent in one or more horizons at a depth between 25 and $75 \mathrm{~cm}$ from the mineral soil surface. So these soils qualified for Dysrudepts at great group level. The soils of P6 under paddy land use have a horizon within $60 \mathrm{~cm}$ of the mineral soil surface, redox depletions with chroma of 2 and also aquic conditions for some time in normal years. So the soils of P6 were classified as Aquic Dysrudepts at subgroup level. Other soils qualified for Typic Dysrudepts at subgroup level.

\section{References}

All India Soil and Land Use Survey Organization (1971) Soil Survey Manual, IARI, New Delhi.

Barshad, I. (1964) Chemistry of soil development. In: Chemistry of the soil. Bear, F. E. (ed.). $2^{\text {nd }}$ Edition. 
Oxford and IBH Publishing Co., New Delhi, pp. 1-70.

Bhaskar, B. P., Mishra, J. P. Baruah, U., Vadivelu, S. Sen, T. K., Butte, P. S. and Dutta, D. P.(2004). Soils on jhum cultivated hill slopes of Narang Kongripara watershed in Meghalaya. Journal of Indian Society of Soil Science 52,125-133.

Bhattacharyya, T., Sen, T.K., Singh, R.S., Nayak, D.C., Sehgal, J. L., (1994). Morphology and classification of Ultisols with Kandic horizon in north eastern region. Journal of the Indian Society of Soil Science 42, 301-306.

Chakraborty, S. K., Singha, H. and Mathur, B. S. (1984) Morphological and physicchemical properties of some alluvial soils of Assam. Journal of the Indian Society of Soil Science 32, 128-136.

Chakravarty, D. N., Sehgal, J. L. and Dev, G. (1978). Influence of climate and topography on the pedogenesis of alluvium derived soils in Assam: Geographical settings, morphological and physico-chemical properties. Indian Journal of Agricultural Chemistry 11, 77-97.

Chakravarty, D. N., Sehgal, J. L. and Dev. G. (1982). Characterization and pedogenic development of Fe-Mn glaebules in the alluvium derived soils of Assam, India. Pedologie 32, 39-51.

Dey, J. K. and Sehgal, J. L. (1997) Characteristics and classification of some alluvial derived paddy and associated non-paddy soils of Assam. Agropedology 7, 22-31.

Dutta, G. (1992a). Characterization and classification of soils of Horticultural Research Station, Kahikuchi, Guwahati, M.Sc.(Agri) thesis, Assam Agric. University, Jorhat

Dutta, S., Karmakar, R. M., and Swargiary, M. M. (2001). Characterization of alluvium derived soils of Brahmaputra valley of Assam. Proceedings of the National Seminar on water and land management including $\mathrm{CAD}$ for socioeconomic upliftment of NE region. Guwahati, Assam. pp.83-93.

Gangopadhyay, S. K. Bhattacharyya, T, and Sonkar, D. (2001). Characterisation and classification of some rubber growing soils of Tripura. Journal of the Indian Society of Soil Science 49, 164-170.

Karmakar, R. M. and Rao, A. E. V. (1994). Pedogenic development of Fe-Mn glaebules in two monadnok soils of Assam. Agropedology 4, 69-74.

Karmakar, R. M. and Rao, A. E. V. (1999a). Soils on different physiographic units in Lower Brahmaputra Valley zone of Assam I. Characterization and classification. Journal of the Indian Society of Soil Science 47, 761-767.

Karmakar, R. M. and Rao, A. E. V. (1999b). Soils on different physiographic units in Lower Brahmaputra Valley zone of Assam II. Sand mineralogy. Journal of the Indian Society of Soil Science 47, 767-770.

Kumar, S. and Naidu, M. V. S. (2012). Characteristics and classification of soils representing major landforms in Vadamalapeta mandal of Chittoor district, Andhra Pradesh. Journal of the Indian Society of Soil Science 60, 63-67.

Mehra, O. P., and Jackson, M. L. (1958) Iron oxide removal from soil and clays by a dithionite-citrate system buffered with sodium bicarbonate. Clays and Clay Minerals 5, 317-327.

Nayak, D.C., Gangopadhyay, S.K., Sarkar, Dipak and Sen, T.K. (2002). Characteristics and classification of some acid soils' of lower Subansiri district of Arunachal Pradesh. Agropedology 12, 112-121.

Patil, P. L. and Dasog, G. S. (1999) Genesis 
and classification of ferruginous soils in western ghat and coastal region of north Karnataka. Agropedology 9, 115.

Tilman D., Cassman, K. G., Matson, P. A., Naylor, R. and Polasky S. (2002). Agricultural sustainability and intensive production practices. Nature 418, 671-677.

Walkley, A. and Black, I. A. (1934) An examination of the Degtjareff method for determining organic carbon in soils: Effect of variations in digestion conditions and of inorganic soil constituents. Soil Science 63, 251263.

Wallenius, T. H., Pennanem, J. and Burton P. J. (2011). Long-term decreasing trend in forest fires in northwestern Canada. Ecosphere 2(5), 53.

\section{How to cite this article:}

Juri Das, R. M. Karmakar, Danish Tamuly, Ingudam Bhupenchandra and Laishram Kanta Singh. 2019. Characterization and Classification of Soils under Lower Brahmaputra Valley of Assam, India. Int.J.Curr.Microbiol.App.Sci. 8(12): 968-981.

doi: https://doi.org/10.20546/ijcmas.2019.812.125 